\title{
Entanglement entropy on the fuzzy sphere
}

\author{
Joanna L. Karczmarek and Philippe Sabella-Garnier \\ Department of Physics and Astronomy, University of British Columbia, \\ 6224 Agricultural Road, Vancouver, Canada \\ E-mail: joanna@phas.ubc.ca, psabella@phas.ubc.ca
}

ABSTRACT: We obtain entanglement entropy on the noncommutative (fuzzy) two-sphere. To define a subregion with a well defined boundary in this geometry, we use the symbol map between elements of the noncommutative algebra and functions on the sphere. We find that entanglement entropy is not proportional to the length of the region's boundary. Rather, in agreement with holographic predictions, it is extensive for regions whose area is a small (but fixed) fraction of the total area of the sphere. This is true even in the limit of small noncommutativity. We also find that entanglement entropy grows linearly with $N$, where $N$ is the size of the irreducible representation of $\mathrm{SU}(2)$ used to define the fuzzy sphere.

Keywords: Matrix Models, Non-Commutative Geometry

ARXIV EPRINT: 1310.8345 


\section{Contents}

1 Introduction 1

2 Noncommutative geometry 4

2.1 Noncommutative plane 4

2.2 Noncommutative sphere $\quad 6$

2.3 The polar cap on the noncommutative sphere 9

$\begin{array}{lll}3 & \text { Results } & 10\end{array}$

$\begin{array}{lll}4 & \text { Discussion and future work } & 14\end{array}$

\section{Introduction}

Field theories on noncommutative spaces are interesting for many reasons, one of them being that these theories are inherently nonlocal and as such might serve as toy models for certain phenomena in quantum gravity. For example, it has been conjectured that black hole horizons scramble information so fast that in the membrane paradigm the horizon of a black hole cannot be modeled by a local theory [1]. It has been suggested that noncommutative theories might in fact be examples of fast scramblers, since (at least at strong coupling) they exhibit enhanced thermalization rates compared to local theories [2].

Noncommutative gauge theories arise naturally in string theory as the effective theories for low energy degrees of freedom on a D-brane with a worldvolume magnetic field [3]. Using this fact, it is possible $[4,5]$ to find holographic duals to such theories. Intriguingly, the dual geometry is an ordinary manifold (though not asymptotically AdS), with noncommutativity encoded in the shape of the holographic dual. Since the dual has ordinary geometry, it can be used to study intrinsically geometric observables such as the geometric entanglement entropy (entanglement entropy associated with some region of space and obtained by tracing out all degrees of freedom residing outside of this region). If we are able to provide an interpretation for such holographically defined geometric observables in the noncommutative field theory, we can study how noncommutative space emerges both from the gravitational dual and the noncommutative algebra on the field theory side. Entanglement entropy is a particularly interesting geometric observable as it provides information on how degrees of freedom at different points are coupled, probing nonlocality of the theory and perhaps teaching us about its scrambling behaviour (for example, it was shown in [6] that possessing extensive entanglement entropy is a necessary condition for scrambling).

Recent work [7, 8] studied holographic entanglement entropy and mutual information, uncovering some interesting properties of these observables in strongly coupled noncommutative gauge theories. ${ }^{1}$ In particular, [8] argued that UV/IR mixing leads to extensive

\footnotetext{
${ }^{1}$ See also an earlier work [9].
} 
(volume-law) holographic entanglement entropy, instead of the more usual area-law behaviour. To interpret these findings within field theory, we must answer the following questions: can one divide the Hilbert space of a field theory on some noncommutative geometry into two components associated with the inside and the outside of some geometric region? If not, what precisely is the meaning of holographic entanglement entropy in field theory, and if yes, is the volume-law behaviour observed through a holographic description a property associated with strong coupling or would it be also seen at weak coupling? This last question is further motivated by the fact that, for example, the enhancement in thermalization timescale mentioned above is not seen in perturbation theory [2].

In the present paper, we shed some light on these issues by considering one of the simplest nontrivial noncommutative field theories: the theory for a free scalar on a noncommutative (or fuzzy) two-sphere. ${ }^{2,3}$ The main advantage of working with the noncommutative sphere is that the field theory is UV-finite and expressible as a finite size matrix model. Further, the matrix model for a free scalar is purely quadratic and therefore entanglement entropy is straightforward to compute.

To obtain the theory of a real scalar on a noncommutative two-sphere, we embed the sphere in three dimensions and represent the three Cartesian coordinates by $\mathrm{SU}(2)$ generators in the $\mathrm{N}$ dimensional irreducible representation $[12,13]$ :

$$
X^{a}=R \frac{L^{a}}{\sqrt{j(j+1)}},\left[L^{a}, L^{b}\right]=i \epsilon^{a b c} L^{c},
$$

where the spin $j=(N-1) / 2$. Since

$$
L^{a} L^{a}=j(j+1)=\frac{N^{2}-1}{4},
$$

the radius of the sphere is $R$. A real scalar field $\varphi$ living on the sphere is described by a hermitian $N \times N$ matrix $\Phi$. Since $L^{a}$ are generators of rotations, derivatives on the sphere are given by commutators with $L^{a}$ and the Laplacian acting on a field $\varphi$ is

$$
-\frac{1}{R^{2}}\left[L^{a},\left[L^{a}, \Phi\right]\right] .
$$

Since trace replaces integration over the sphere

$$
R^{2} \int \sin (\theta) d \theta d \phi \varphi(\theta, \phi) \rightarrow \frac{4 \pi R^{2}}{N} \operatorname{Tr} \Phi,
$$

our free field has the following Hamiltonian

$$
H=\frac{4 \pi R^{2}}{N} \frac{1}{2} \operatorname{Tr}\left(\dot{\Phi}^{2}-R^{-2}\left[L_{i}, \Phi\right]^{2}+\mu^{2} \Phi^{2}\right) .
$$

The theory has one free dimensionless parameter, mass measured in units of the radius, or $m=R \mu$.

\footnotetext{
${ }^{2}$ The theory of a free scalar on a noncommutative plane is equivalent to the free scalar on a commutative plane and therefore not interesting.

${ }^{3}$ Previous work on entanglement entropy on a fuzzy sphere includes [10, 11], where the entanglement entropy for half the sphere was computed.
} 
In this paper, we obtain entanglement entropy associated with a polar cap region $C$ whose size is controlled by a polar angle $\theta$ (see figure 1). Using a map from operators (matrices) to functions on the sphere - called a symbol [13] — we determine which entries of the matrix $\Phi$ correspond to degrees of freedom inside this polar cap and which correspond to the outside. Thus, we write the Hilbert space $\mathcal{H}$ of our matrix model as a product of two smaller spaces: one corresponding to the inside of the polar cap, $\mathcal{H}_{C}$ and one corresponding to the outside of the polar cap, $\mathcal{H}_{\bar{C}}$, with $\mathcal{H}=\mathcal{H}_{C} \otimes \mathcal{H}_{\bar{C}}$. We compute entanglement entropy using the usual definition:

$$
S=-\operatorname{Tr}_{C}\left(\rho_{C} \ln \rho_{C}\right)
$$

where

$$
\rho_{c}=\operatorname{Tr}_{\bar{C}}|\psi\rangle\langle\psi|
$$

is the density matrix associated with $\mathcal{H}_{c}$ when the entire quantum system is in a state $|\psi\rangle$ (which we will take to be the vacuum).

Of course, it is not possible to draw a sharp boundary for a region on a noncommutative sphere. For our procedure, the boundary of the region $C$ can be thought of as having a thickness of $\sqrt{\boldsymbol{\theta}}$ where $\boldsymbol{\theta}=R^{2} / N$ is the noncommutativity parameter. We can compare the length-scale $\sqrt{\boldsymbol{\theta}}=R / \sqrt{N}$ to the the UV cutoff of the theory, $\epsilon$. The UV cutoff is most easily obtained by dividing the area of the sphere $4 \pi R^{2}$ by the total number of degrees of freedom in our noncommutative model, $N^{2}$. Since a small region of area $\epsilon^{2}$ should contain exactly one degree of freedom, $\epsilon$ is approximately $R / N$. We see that $\sqrt{\boldsymbol{\theta}}$ is parametrically larger than $\epsilon$.

Since, in the large $N$ limit, the noncommutative sphere is supposed to reduce to the commutative one, the reader might expect entanglement entropy on a noncommutative sphere to agree with that on a commutative sphere for regions whose diameter is larger than $\sqrt{\boldsymbol{\theta}}$. If that were the case, we would not be able to discover any deviation in the noncommutative case, as regions whose boundary has thickness $\sqrt{\boldsymbol{\theta}}$ cannot be smaller than $\sqrt{\boldsymbol{\theta}}$. Fortunately, as has been observed in $[7,8]$, strong deviations from commutative behaviour should be seen in entanglement entropy for regions whose size is of order $\boldsymbol{\theta} / \epsilon=R$, which corresponds to the entire sphere (or, equivalently, the IR cutoff of our theory). The reason why noncommutative entanglement entropy for regions larger than $\sqrt{\boldsymbol{\theta}}$ (but smaller than $\boldsymbol{\theta} / \epsilon)$ does not agree with its commutative counterpart lies in UV/IR mixing: because of this mixing, a noncommutative theory with a UV cutoff $\epsilon$ is expected to have nonlocal behaviour up to a length-scale $\boldsymbol{\theta} / \epsilon[14]{ }^{4}$ Thus, we expect deviation from commutative behaviour at least for regions whose area is a small (but finite in the commutative limit) fraction of the total sphere area. Any such deviation we see can be interpreted as a result of UV/IR mixing on the noncommutative sphere.

\footnotetext{
${ }^{4}$ In [14], the UV/IR connection was studied on the noncommutative plane. The UV/IR mixing on the fuzzy sphere has been studied using the one-loop effective action in several interacting theories (see for example [15] and [16]). Here we simply use the results from flat noncommutative geometry as a guide to interpreting our results. Notice that entanglement entropy could potentially be sensitive to UV/IR mixing not detected by, for example, divergences in the two-point functions. In this case our results could be interpreted as evidence of previously undiscovered UV/IR mixing.
} 
In fact, this is precisely what we discover: for small regions, entanglement entropy on the noncommutative sphere grows linearly with the area of the region (and not with the length of its boundary), and hence follows the volume law. ${ }^{5}$ For regions whose area is comparable to the total area of the sphere, the entanglement entropy receives higher power corrections. However, while in [8] it was shown that the entanglement entropy for a field theory with some effective noncommutativity scale $a_{\theta}$ at strong coupling undergoes a phase transition between volume-law at length-scales below $a_{\theta}^{2} / \epsilon$ and area-law at lengthscales above that, on the noncommutative sphere there is no such phase transition. This is due to the compactness of the manifold and the resulting IR cutoff which was absent in the holographic calculation. The phase transition is replaced with crossover behaviour near the IR cutoff (for a region whose size is half that of the whole sphere) and the higher power corrections mentioned above lead to a smooth behaviour.

The rest of this paper is organized as follows: in section 2 we present a self-contained review of symbol maps and star products on the noncommutative plane and the noncommutative sphere, before presenting our proposal for how to define the polar cap region in the noncommutative sphere geometry in section 2.3. Our numerical methodology and results follow in section 3. Finally, in section 4 we provide possible interpretations for our results and discuss open problems for future work.

\section{Noncommutative geometry}

In this section, we review the concepts of a symbol and a corresponding star product as a way to encode the noncommutative structure of geometry. We begin by reviewing the better-known example of a noncommutative plane, and then show how the same tools can be applied to treat the noncommutative sphere. Our general approach is similar to that in [17], though the details are somewhat different. In section 2.3 we use our symbol map on the fuzzy sphere to obtain our desired mapping between the polar cap and matrix elements.

\subsection{Noncommutative plane}

The noncommutative plane has as its structure algebra the Heisenberg algebra. This is the algebra generated by two operators $\hat{x}$ and $\hat{y}$ with the commutation relation

$$
[\hat{x}, \hat{y}]=i \boldsymbol{\theta}
$$

$\sqrt{\boldsymbol{\theta}}$ has the units of length and is the fundamental length-scale of noncommutativity.

A common treatment of noncommutative geometry uses a map $s$ which takes elements $\hat{A}$ of the structure algebra to functions on the corresponding commutative manifold (in this case, the ordinary two-dimensional plane), $s(\hat{A})=f_{A}(x, y)$. The function $f_{A}$ is called the symbol of the algebra element $\hat{A}$. The symbol map is not unique: there are many different

\footnotetext{
${ }^{5}$ Even though we are working in two spacial dimensions, we will continue to use higher-dimensional terminology and refer to entropy growing with the area of the region as volume-law behaviour and entanglement entropy proportional to the length of the boundary of the region as area-law behaviour.
} 
definitions of $s$, corresponding to different ways to order the algebra element $s^{-1}\left(f_{A}\right)$. For every symbol map $s$ there exists a so-called star product $*$ with the property that

$$
s(\hat{A} \hat{B})=s(\hat{A}) * s(\hat{B}) .
$$

As $\hat{A} \hat{B} \neq \hat{B} \hat{A}, *$ cannot be a commutative product, but it is associative.

The most often used symbol map is the Weyl-ordered symbol, which leads to the Moyal star product. Explicitly, these are

$$
s_{W}(\hat{A})=\frac{1}{(2 \pi)^{2}} \int d^{2} k \operatorname{Tr}\left(e^{i k \cdot(x-\hat{x})} \hat{A}\right)
$$

and

$$
(f \star g)(x, y)=\left.e^{\frac{i}{2} \boldsymbol{\theta}\left(\frac{\partial}{\partial \xi_{1}} \frac{\partial}{\partial \zeta_{2}}-\frac{\partial}{\partial \zeta_{1}} \frac{\partial}{\partial \xi_{2}}\right)} f\left(x+\xi_{1}, y+\zeta_{1}\right) g\left(x+\xi_{2}, y+\zeta_{2}\right)\right|_{\xi_{1}=\zeta_{1}=\xi_{2}=\zeta_{2}=0}
$$

However, this is not the treatment we wish to present. Instead, we follow the approach due to Berezin [18] and define the symbol as an expectation value in a coherent state.

Accordingly, let us define the raising and lowering operators, $\hat{a}=\hat{x}+i \hat{y}, \hat{a}^{\dagger}=\hat{x}-i \hat{y}$, with $\left[\hat{a}, \hat{a}^{\dagger}\right]=2 \boldsymbol{\theta}$, as well as the canonical coherent states which are eigenstates of the lowering operator, $\hat{a}|\alpha\rangle=\alpha|\alpha\rangle$. We can think of each coherent state $|\alpha\rangle$ as corresponding to the point $(x, y)=(\operatorname{Re}(\alpha), \operatorname{Im}(\alpha))$ since $\langle\alpha|\hat{x}| \alpha\rangle=\operatorname{Re}(\alpha)$ and $\langle\alpha|\hat{y}| \alpha\rangle=\operatorname{Im}(\alpha)$. The overlap between these coherent states decreases rapidly as the corresponding points are separated: $|\langle\beta \mid \alpha\rangle|=e^{-|\alpha-\beta|^{2} / 4 \boldsymbol{\theta}}$, with a length-scale controlled by the noncommutativity scale $\sqrt{\boldsymbol{\theta}}$. The Berezin symbol is defined as

$$
s(\hat{A})=f_{A}(\alpha, \bar{\alpha})=\langle\alpha|\hat{A}| \alpha\rangle .
$$

The Berezin symbol corresponds to normal ordering, in contrast with the Weyl symbol which was based on the symmetric ordering.

To derive a star product compatible with the Berezin symbol, we need to use the fact that $|\alpha\rangle$ is, up to a normalization factor, a holomorphic function of the complex variable $\alpha$ :

$$
|\alpha\rangle=e^{-|\alpha|^{2} / 4 \boldsymbol{\theta}} \sum_{n=0}^{\infty} \frac{(\alpha / \sqrt{2 \boldsymbol{\theta}})^{n}}{\sqrt{n !}}|n\rangle .
$$

This implies that $\langle\beta|A| \alpha\rangle /\langle\beta \mid \alpha\rangle$ is holomorphic in $\alpha$ and antiholomorphic in $\beta$. Thus, since $\langle\beta \mid \beta\rangle=1$, we have

$$
\frac{\langle\beta|A| \alpha\rangle}{\langle\beta \mid \alpha\rangle}=e^{-\beta \frac{\partial}{\partial \alpha}} \frac{\langle\beta|A| \beta+\alpha\rangle}{\langle\beta \mid \beta+\alpha\rangle}=e^{-\beta \frac{\partial}{\partial \alpha}} e^{\alpha \frac{\partial}{\partial \beta}} \frac{\langle\beta|A| \beta\rangle}{\langle\beta \mid \beta\rangle}=e^{-\beta \frac{\partial}{\partial \alpha}} e^{\alpha \frac{\partial}{\partial \beta}} f_{A}(\beta, \bar{\beta}),
$$

and, similarly,

$$
\frac{\langle\alpha|A| \beta\rangle}{\langle\alpha \mid \beta\rangle}=e^{-\bar{\beta} \frac{\partial}{\partial \bar{\alpha}}} e^{\bar{\alpha} \frac{\partial}{\partial \beta}} f_{A}(\beta, \bar{\beta}) .
$$


We can now compute an explicit expression for the star product, using the completeness relation for coherent states:

$$
\begin{aligned}
\left(f_{A} * f_{B}\right)(\beta, \bar{\beta}) & =\langle\beta|A B| \beta\rangle=\frac{1}{2 \pi \boldsymbol{\theta}} \int d^{2} \alpha\langle\beta|A| \alpha\rangle\langle\alpha|B| \beta\rangle \\
& =\frac{1}{2 \pi \boldsymbol{\theta}} \int d^{2} \alpha|\langle\beta \mid \alpha\rangle|^{2}\left[e^{-\beta \frac{\partial}{\partial \alpha}} e^{\alpha \frac{\partial}{\partial \beta}} f_{A}(\beta, \bar{\beta})\right] \quad\left[e^{-\bar{\beta} \frac{\partial}{\partial \bar{\alpha}} e^{\bar{\alpha} \frac{\partial}{\partial \beta}}} f_{B}(\beta, \bar{\beta})\right] \\
& =\frac{1}{2 \pi \boldsymbol{\theta}} \int d^{2} \alpha\left(e^{\beta \frac{\partial}{\partial \alpha}+\bar{\beta} \frac{\partial}{\partial \bar{\alpha}}}|\langle\beta \mid \alpha\rangle|^{2}\right)\left[e^{\alpha \frac{\partial}{\partial \beta}} f_{A}(\beta, \bar{\beta})\right] \quad\left[e^{\bar{\alpha} \frac{\partial}{\partial \beta}} f_{B}(\beta, \bar{\beta})\right] \\
& =\frac{1}{2 \pi \boldsymbol{\theta}} \int d^{2} \alpha|\langle\beta \mid \alpha+\beta\rangle|^{2}\left[e^{\alpha \frac{\partial}{\partial \beta}} f_{A}(\beta, \bar{\beta})\right] \quad\left[e^{\bar{\alpha} \frac{\partial}{\partial \bar{\beta}}} f_{B}(\beta, \bar{\beta})\right] \\
& =\frac{1}{2 \pi \boldsymbol{\theta}} \int d^{2} \alpha e^{-|\alpha|^{2} / 2 \boldsymbol{\theta}}\left[e^{\alpha \frac{\partial}{\partial \beta}} f_{A}(\beta, \bar{\beta})\right]\left[e^{\bar{\alpha} \frac{\partial}{\partial \bar{\beta}}} f_{B}(\beta, \bar{\beta})\right] \\
& =\left.e^{2 \boldsymbol{\theta} \frac{\partial}{\partial \zeta} \frac{\partial}{\partial \bar{\eta}}} f_{A}(\beta+\zeta, \bar{\beta}) f_{B}(\beta, \bar{\beta}+\bar{\eta})\right|_{\zeta=\bar{\eta}=0}
\end{aligned}
$$

This is known as the Vorol product. The Vorol product is equivalent to the more commonly used Moyal star product shown above.

\subsection{Noncommutative sphere}

A rather similar approach allows us to study the noncommutative sphere. The structure algebra is simply the algebra of $N \times N$ hermitian matrices, $M_{n}$. In this algebra, as we have already discussed in the Introduction, we single out three matrices $L^{a}$ satisfying the $\mathrm{SU}(2)$ commutation relations. Since the $L^{a}$ form an irreducible representation of SU(2), these three matrices generate all of $M_{n}$.

We will use as a basis the eigenvectors of the $L^{3}$ angular momentum, $|m\rangle$ :

$$
L^{3}|m\rangle=m|m\rangle, \quad m=-j \ldots j, \quad\langle m \mid m\rangle=1, \quad j=\frac{N-1}{2} .
$$

To define an analog of the coherent state, ${ }^{6}$ let $\hat{n}$ be a unit 3 -vector (or a point on a unit sphere). Then, define $L_{\hat{n}}=\hat{n}^{a} L^{a}$. A coherent state at point $R \hat{n}$ on the sphere of radius $R$ is then $|\hat{n}\rangle$, where

$$
L_{\hat{n}}|\hat{n}\rangle=j|\hat{n}\rangle, \quad\langle\hat{n} \mid \hat{n}\rangle=1 .
$$

The coherent state at the north pole is the state with the largest angular momentum in the 3-direction; a coherent state at any other point can be obtained from the one at the north pole by a $\mathrm{SU}(2)$ rotation. Recall the Wigner formula, ${ }^{7}$

$$
|\langle m \mid \hat{n}\rangle|=\sqrt{\frac{(2 j) !}{(j+m) !)(j-m) !}}\left(\cos \frac{\theta}{2}\right)^{j+m}\left(\sin \frac{\theta}{2}\right)^{j-m}
$$

where $\theta$ is the polar angle at point $\hat{n}$ on the unit sphere: the angle between the positive 3 -axis and $\hat{n}$.

\footnotetext{
${ }^{6}$ For another approach to coherent states on the fuzzy sphere, see [19].

${ }^{7}$ See for example [20] equation (3.8.33).
} 
One consequence is that, if the angle between two unit vectors $\hat{n}_{1}$ and $\hat{n}_{2}$ is $\chi=$ $\arccos \left(\hat{n}_{1} \cdot \hat{n}_{2}\right)$, then

$$
\left|\left\langle\hat{n}_{1} \mid \hat{n}_{2}\right\rangle\right|=\left(\cos \frac{\chi}{2}\right)^{2 j}=\left(\frac{\hat{n}_{1} \cdot \hat{n}_{2}+1}{2}\right)^{j} .
$$

For large $j$, the overlap between the states $\left|\hat{n}_{1}\right\rangle$ and $\left|\hat{n}_{2}\right\rangle$ decreases sharply as the angle between them is increased. If we let $\chi=2 / \sqrt{j}$, we have

$$
\left(\cos \frac{\chi}{2}\right)^{2 j} \approx\left(1-\frac{1}{2 j}\right)^{2 j}
$$

which approaches $1 / e$ for large $j$. Thus, the effective width of the coherent states on a sphere of radius $\mathrm{R}$ is proportional to $R N^{-1 / 2}$. A single coherent state covers an area proportional to $R^{2} / N$, which is natural given that the noncommutative sphere should contain $N$ unit noncommutative 'cells'. ${ }^{8}$

It is easy to convince oneself that for $\hat{n}$ in the 1-3 plane, $\langle m \mid \hat{n}\rangle$ can be real when we take $L_{2}$ to be purely imaginary (and therefore antisymmetric). To restore the phase of $\langle m \mid \hat{n}\rangle$ for all directions $\hat{n}$, write $\hat{n}$ in polar coordinates: $\hat{n}=(\sin \theta \cos \phi, \sin \theta \sin \phi, \cos \theta)$. As we just discussed, for the azimuthal angle $\phi=0,\langle m \mid \hat{n}\rangle$ is real. For all other angles, we rotate around the 3 -axis to obtain

$$
\langle m \mid \hat{n}\rangle=\sqrt{\frac{(2 j) !}{(j+m) !)(j-m) !}}\left(\frac{1}{2} \sin \theta\right)^{j}\left(\tan \frac{\theta}{2}\right)^{-m} e^{-i m \phi} .
$$

Now, consider a complex variable $\alpha=R \tan (\theta / 2) e^{i \phi}$. This is simply the complex coordinate arising from a stereoscopic projection. This coordinate does not cover the entire sphere (it is singular at the point $\theta=\pi$ ), but a complementary complex coordinate, $\tilde{\alpha}=R \tan ((\pi-\theta) / 2) e^{-i \phi}$, does. Since $\tilde{\alpha}(\alpha)=R^{2} / \alpha$ is a holomorphic function, together these two complex coordinates define a complete complex structure.

We will now change notation and denote the coherent states with $|\alpha\rangle$ instead of $|\hat{n}\rangle$. Just as it was with the coherent state on the plane, up to a normalization factor our coherent states on the sphere are holomorphic in the complex variable $\alpha$.

$$
|\alpha\rangle=\left(\frac{1}{2} \sin \theta\right)^{j} \sum_{m=-j}^{j} \sqrt{\frac{(2 j) !}{(j+m) !)(j-m) !}}\left(\frac{\alpha}{R}\right)^{-m}|m\rangle .
$$

These coherent states are overcomplete

$$
\frac{N}{4 \pi R^{2}} \int \frac{4 d^{2} \alpha}{\left(1+|\alpha / R|^{2}\right)^{2}}|\alpha\rangle\langle\alpha|=1
$$

with respect to the $\mathrm{SU}(2)$ invariant measure on the sphere, $\frac{4 d^{2} \alpha}{\left(1+|\alpha / R|^{2}\right)^{2}}$.

\footnotetext{
${ }^{8}$ In string theory, we would say that the spherical D2-brane has $N$ units of flux piercing it, corresponding to $N$ D0-branes dissolved in its worldvolume. The effective theory describing $N$ D0-branes is written in terms of $N \times N$ hermitian matrices, lending the D2-brane the noncommutative structure we are studying.
} 
For any matrix operator $A$ in $M_{n}$, consider its Berezin symbol $f_{A}(\alpha)=\langle\alpha|A| \alpha\rangle$. The Berezin symbol is a function on the sphere which corresponds to the matrix in $M_{n}$. Since equation (2.7) is valid on the sphere (as it relies only on the coherent states being holomorphic), we have:

$$
\begin{aligned}
\left(f_{A} * f_{B}\right)(\beta, \bar{\beta})= & \langle\beta|A B| \beta\rangle=\frac{N}{\pi R^{2}} \int \frac{d^{2} \alpha}{\left(1+|\alpha / R|^{2}\right)^{2}}\langle\beta|A| \alpha\rangle\langle\alpha|B| \beta\rangle \\
= & \frac{N}{\pi R^{2}} \int \frac{d^{2} \alpha}{\left(1+|\alpha / R|^{2}\right)^{2}}|\langle\beta \mid \alpha\rangle|^{2} \\
& \times\left[e^{-\beta \frac{\partial}{\partial \alpha}} e^{\alpha \frac{\partial}{\partial \beta}} f_{A}(\beta, \bar{\beta})\right]\left[e^{-\bar{\beta} \frac{\partial}{\partial \bar{\alpha}} e^{\bar{\alpha}} \frac{\partial}{\partial \bar{\beta}}} f_{B}(\beta, \bar{\beta})\right] .
\end{aligned}
$$

To simplify our computation, we will compute only the star product at the north pole, $\beta=0$. We have

$$
\begin{aligned}
\left(f_{A} * f_{B}\right)(0,0)= & \frac{N}{\pi R^{2}} \int \frac{d^{2} \alpha}{\left(1+|\alpha / R|^{2}\right)^{2}}|\langle 0 \mid \alpha\rangle|^{2}\left[e^{\alpha \frac{\partial}{\partial \beta}} f_{A}(\beta, \bar{\beta})\right]_{\beta=0}\left[e^{\bar{\alpha} \frac{\partial}{\partial \beta}} f_{B}(\beta, \bar{\beta})\right]_{\beta=0} \\
= & \frac{N}{\pi R^{2}} \int \frac{d^{2} \alpha}{\left(1+|\alpha / R|^{2}\right)^{2}}\left(\frac{1}{1+|\alpha / R|^{2}}\right)^{2 j} \\
& \quad \times\left[e^{\alpha \frac{\partial}{\partial \beta}} f_{A}(\beta, \bar{\beta})\right]_{\beta=0}\left[e^{\bar{\alpha} \frac{\partial}{\partial \beta}} f_{B}(\beta, \bar{\beta})\right]_{\beta=0}
\end{aligned}
$$

Now, on the surface of it, this integral does not appear convergent; however, we have:

$$
\left.\frac{\partial^{p}}{\partial \beta^{p}} f(\beta, \bar{\beta})\right|_{\beta=0}=0 \quad \text { for } p>2 j
$$

To see that this is the case, just write the Berezin symbol for any operator $A$ as

$$
\begin{aligned}
f_{A}(\beta, \bar{\beta}) & =\sum_{n, m=-j}^{j}\langle\beta \mid n\rangle\langle n|A| m\rangle\langle m \mid \beta\rangle \\
& =\sum_{n, m=-j}^{j} \frac{(2 j) !\langle n|A| m\rangle}{\sqrt{(j+m) !(j+n) !(j-n) !(j-m) !}} \frac{\beta^{j-m} \bar{\beta}^{j-n}}{(1+\beta \bar{\beta})^{2 j}}
\end{aligned}
$$

$\frac{\partial^{p}}{\partial \beta^{p}}$ acting on a $(n, m)$ term in the above sum is nonzero only if $p=j-m$ and $n=j$. Thus, $p$ is at most $2 j$.

Returning to our expression for the star product, we now have that

$$
\begin{aligned}
\left(f_{A} * f_{B}\right)(0,0) & =\frac{N}{\pi R^{2}} \sum_{p, q=0}^{2 j} \frac{1}{p ! q !} \int \frac{d^{2} \alpha \alpha^{p} \bar{\alpha}^{q}}{\left(1+|\alpha / R|^{2}\right)^{2 j+2}}\left[\frac{\partial^{p}}{\partial \beta^{p}} f_{A}(\beta, \bar{\beta})\right]_{\beta=0}\left[\frac{\partial^{q}}{\partial \bar{\beta}^{q}} f_{B}(\beta, \bar{\beta})\right]_{\beta=0} \\
& =N \sum_{p=0}^{2 j} R^{2 p} \frac{(2 j-p) !}{p !(2 j+1) !}\left[\frac{\partial^{p}}{\partial \beta^{p}} f_{A}(\beta, \bar{\beta})\right]_{\beta=0}\left[\frac{\partial^{p}}{\partial \bar{\beta}^{p}} f_{B}(\beta, \bar{\beta})\right]_{\beta=0} \\
& =\sum_{p=0}^{2 j} \frac{(2 j-p) !}{p !(2 j) !}\left[R^{2 p} \frac{\partial^{p}}{\partial \alpha^{p}} \frac{\partial^{p}}{\partial \bar{\beta}^{p}} f_{A}(\alpha, \bar{\alpha}) f_{B}(\beta, \bar{\beta})\right]_{\alpha=\beta=0}
\end{aligned}
$$



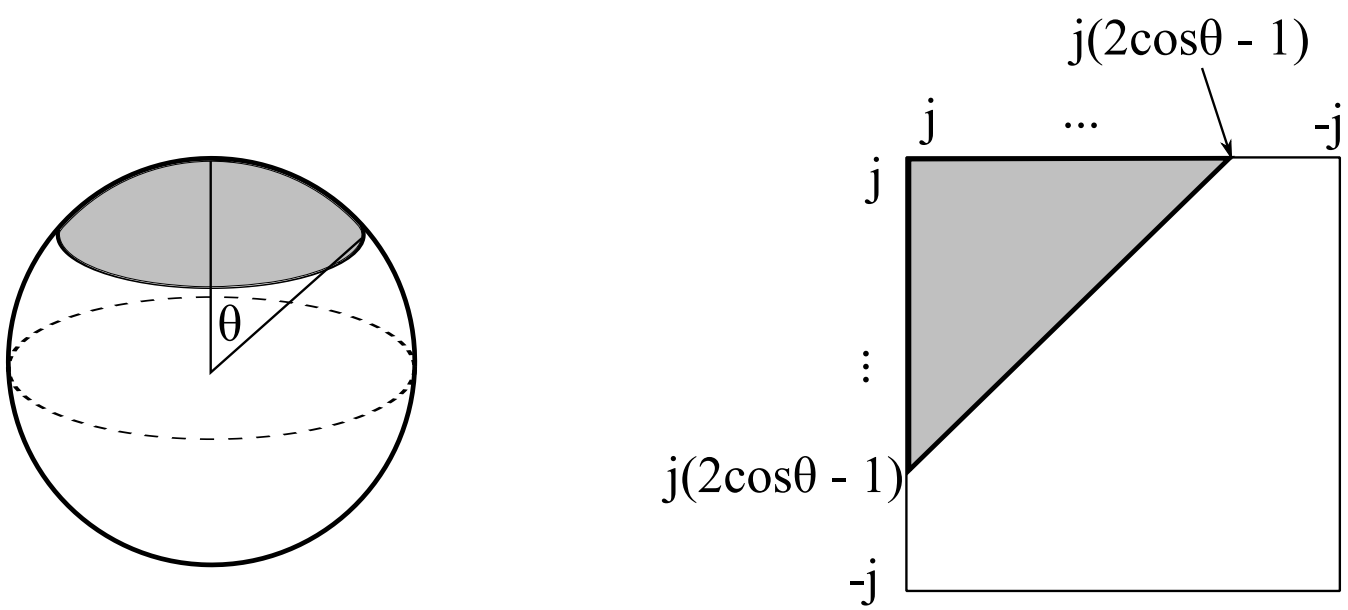

Figure 1. Degrees of freedom on the sphere and their matrix counterparts.

This is the star product derived and used, for example, in [21].

If the functions $f_{A}$ and $f_{B}$ are very smooth, only the first few terms will contribute. We can then write

$$
\begin{aligned}
\left(f_{A} * f_{B}\right)(0,0) & \approx \sum_{p=0} \frac{(2 j)^{-p}}{p !}\left[R^{2 p} \frac{\partial^{p}}{\partial \alpha^{p}} \frac{\partial^{p}}{\partial \bar{\beta}^{p}} f_{A}(\alpha, \bar{\alpha}) f_{B}(\beta, \bar{\beta})\right]_{\alpha=\beta=0} \\
& =\left.e^{\frac{R^{2}}{2 j} \frac{\partial}{\partial \alpha} \frac{\partial}{\partial \beta}} f_{A}(\alpha, \bar{\alpha}) f_{B}(\beta, \bar{\beta})\right|_{\alpha=\beta=0},
\end{aligned}
$$

which reduces to the Vorov product with the noncommutativity parameter $R^{2} /(4 j)$.

\subsection{The polar cap on the noncommutative sphere}

Now, consider an operator $\left|m_{1}\right\rangle\left\langle m_{2}\right|$ and its Berezin symbol $f_{m_{1}, m_{2}}(\alpha)=\left\langle\alpha \mid m_{1}\right\rangle\left\langle m_{2} \mid \alpha\right\rangle$. We have

$$
\begin{aligned}
& f_{m_{1}, m_{2}}=(\text { phase }) \frac{(2 j) !}{\sqrt{\left.\left.\left(j+m_{1}\right) !\right)\left(j-m_{1}\right) !\left(j+m_{2}\right) !\right)\left(j-m_{2}\right) !}} \\
& \times\left(\cos \frac{\theta}{2}\right)^{2 j+\left(m_{1}+m_{2}\right)}\left(\sin \frac{\theta}{2}\right)^{2 j-\left(m_{1}+m_{2}\right)}
\end{aligned}
$$

For large $j$, the function

$$
\left(\cos \frac{\theta}{2}\right)^{2 j(1+x)}\left(\sin \frac{\theta}{2}\right)^{2 j(1-x)}
$$

has a sharp peak at $\theta$ such that $\cos \theta=x$. Therefore the Berezin symbol $f_{m_{1}, m_{2}}(\alpha)$ is largest when the vector $\hat{n}$ makes an angle $\theta_{0}=\arccos \left(\frac{m_{1}+m_{2}}{2 j}\right)$ with the vertical axis. For $\theta$ close to $\theta_{0}$, we can write

$$
\left(\cos \frac{\theta}{2}\right)^{2 j(1+x)}\left(\sin \frac{\theta}{2}\right)^{2 j(1-x)} \approx\left[\frac{1}{4}(1+x)^{1+x}(1-x)^{1-x}\right]^{j} e^{-j\left(\theta-\theta_{0}\right)^{2}} .
$$


Therefore, the Berezin symbol of the operator $\left|m_{1}\right\rangle\left\langle m_{2}\right|$ is appreciable only when the polar angle $\theta$ is within $1 / \sqrt{j}$ of $\theta_{0}$.

This implies that the degrees of freedom corresponding to a polar cap $C$ of angular radius $\theta$ (i.e., all points on the sphere whose polar angle is less than $\theta$ ) can be identified, in the large $j$ limit, with the set of matrix elements $\left\{\left\langle m_{1}|\Phi| m_{2}\right\rangle \mid m_{1}+m_{2}>2 j \cos (\theta)\right\}$. In particular, to compute the entanglement entropy for half the sphere, we should include the degrees of freedom in 'half' the matrix. This was conjectured, but not proven, in [10]. Note that it does not matter whether the (anti)diagonal degrees of freedom are included or not, as the answer will be the same in any pure state.

Since our coherent states have a width proportional to $R / \sqrt{N}$, the boundary of our polar cap region $C$ can be thought as having a thickness of the same size, $R / \sqrt{N}$. In other words, if we consider the subspace of $M_{n}$ spanned just by the matrix elements indicated in figure 1, the corresponding functions on the sphere would have support on the polar cap $C$ and Gaussian drop-off 'tails' controlled by $R / \sqrt{N}$ outside of the polar cap $C$.

Since the full set of symbols given by equation (2.24) have wavelengths as short as $R / j$ (natural, given that the UV cutoff $\epsilon=R / N$ ), one can wonder whether it is possible to fine-tune our procedure to produce a region with a 'thinner' boundary. We leave this for future work.

\section{Results}

Since our Hamiltonian (1.5) is quadratic, we use the formalism developed in [22] to numerically compute entanglement entropy.

We label the entries of $\Phi$ as $^{9}$

$$
\Phi=\left(\begin{array}{ccccc}
\Phi_{1} & \frac{\Phi_{2}+i \Phi_{3}}{\sqrt{2}} & \frac{\Phi_{4}+i \Phi_{5}}{\sqrt{2}} & \frac{\Phi_{7}+i \Phi_{8}}{\sqrt{2}} & \ldots \\
\frac{\Phi_{2}-i \Phi_{3}}{\sqrt{2}} & \Phi_{6} & \frac{\Phi_{9}+i \Phi_{10}}{\sqrt{2}} & \ldots & \ldots \\
\frac{\Phi_{4}-i \Phi_{5}}{\sqrt{2}} & \frac{\Phi_{9}-i \Phi_{10}}{\sqrt{2}} & \ldots & \ldots & \ldots \\
\frac{\Phi_{7}-i \Phi_{8}}{\sqrt{2}} & \ldots & \ldots & \ldots & \ldots \\
\ldots & \ldots & \ldots & \ldots & \ldots
\end{array}\right)
$$

so that the Hamiltonian (1.5) takes the form

$$
H=\frac{2 \pi}{N} \sum_{a, b=1}^{N^{2}}\left(\pi_{a} \delta_{a b} \pi_{b}+\Phi_{a} K_{a b} \Phi_{b}\right),
$$

where $K$ is the real symmetric positive-definite dynamical matrix

$$
K_{a b}=-\frac{1}{2} \frac{\partial^{2} \operatorname{Tr}\left(\left[L_{i}, \Phi\right]^{2}\right)}{\partial \Phi_{a} \partial \Phi_{b}}+m^{2} \delta_{a b}
$$

and $\pi_{a}=R \dot{\Phi}_{a}$ are canonical momenta.

\footnotetext{
${ }^{9}$ We have chosen the precise computational method we are about to describe for its conceptual simplicity. The parametrization developed in [10] is a more efficient (numerically) approach for this problem. The two approaches gave the same answers.
} 


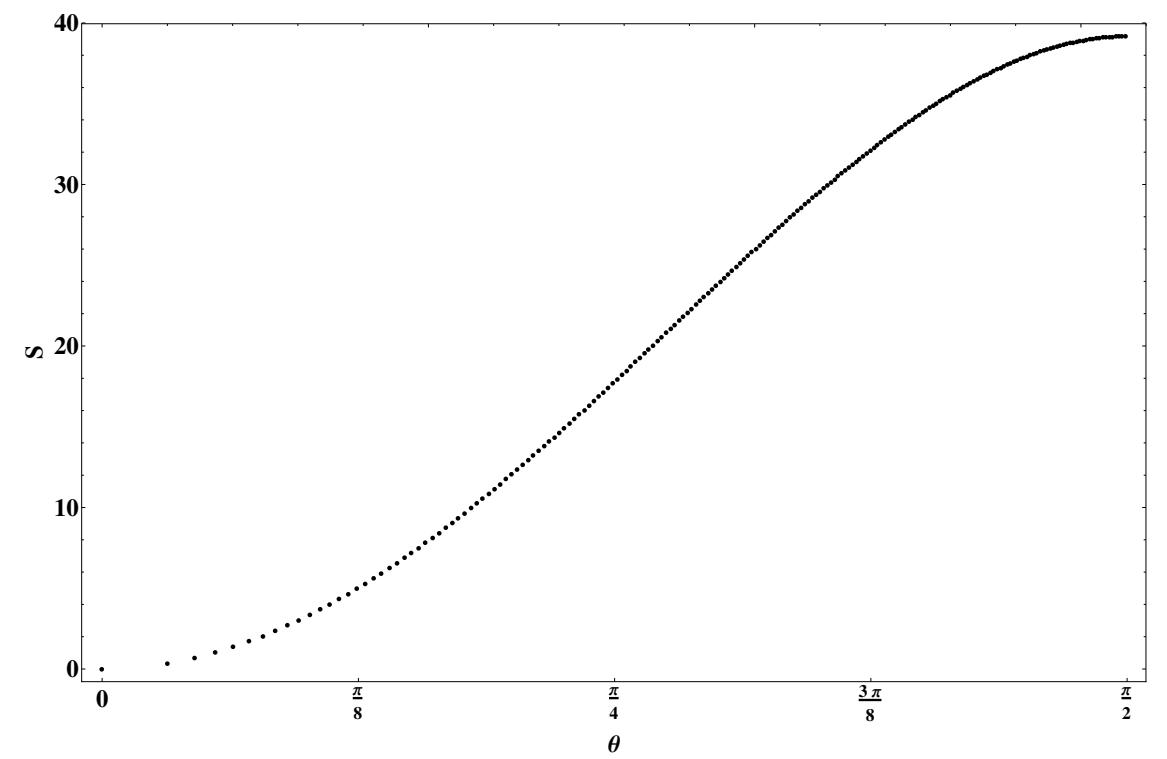

Figure 2. Entanglement entropy $S$ as a function of angular size $\theta$ of polar cap $C$. $N=200$ and $m=1$.

Note that if $m=0, K$ has a zero eigenvalue associated with the matrix $\Phi$ being proportional to the identity. This flat direction leads to infinite entanglement entropy. To study the massless case, one could impose a tracelessness condition on $\Phi$, thus eliminating the massless mode.

To calculate the entanglement entropy associated with a polar cap we must compute the entanglement entropy associated with tracing over Hilbert spaces of oscillators $\Phi_{a}$ in the first $k$ (anti)diagonal 'lines', with $k=2 j(1-\cos \theta)$. Therefore, we need to find the entanglement entropy of the first $n=1+2+3+\ldots+k$ oscillators $(a=1, \ldots, n)$, which we can accomplish by following the procedure outlined in [22]. Since there is a bit of ambiguity in the relationship between $\theta$ and $\mathrm{k}(\theta$ can be shifted by an amount order $1 / N)$, we use this to adjust the correspondence between $\theta$ and $k$ so that the physical condition $S(\theta)=S(\pi-\theta)$ is satisfied. This is possible because entanglement entropies for $n=k(k+1) / 2$ and $n=N^{2}-k(k+1) / 2$ are the same (a good check on our numerics). Thus

$$
\cos \theta=1-\frac{k}{N-\frac{1}{2}}, \text { for } k=1, \ldots, N-1 .
$$

Figure 2 shows entanglement entropy for a polar cap region as a function of the polar angle $\theta$ for $m=1$ and $N=200$. Angles beyond $\theta=\pi / 2$ are not shown as entanglement entropy necessarily has $S(\theta)=S(\pi-\theta)$ for a pure state such as the vacuum. The most interesting feature is the small angle behaviour: $S \sim \theta^{2}$. Notice also that $S(\theta)$ is smooth as a function of $\theta$, including at $\theta=\pi / 2$, indicating that there is no phase transition in the entanglement entropy. Such a phase transition was observed for $\mathcal{N}=4$ Yang-Mills in [7, 8], but here it is absent, probably due to the theory being defined on a compact manifold.

In figure 3 we show the dependence of the entropy $S$ for a half-sphere $(\theta=\pi / 2)$ as a function of $N$. The behaviour is clearly linear (though with an offset dependent on the 


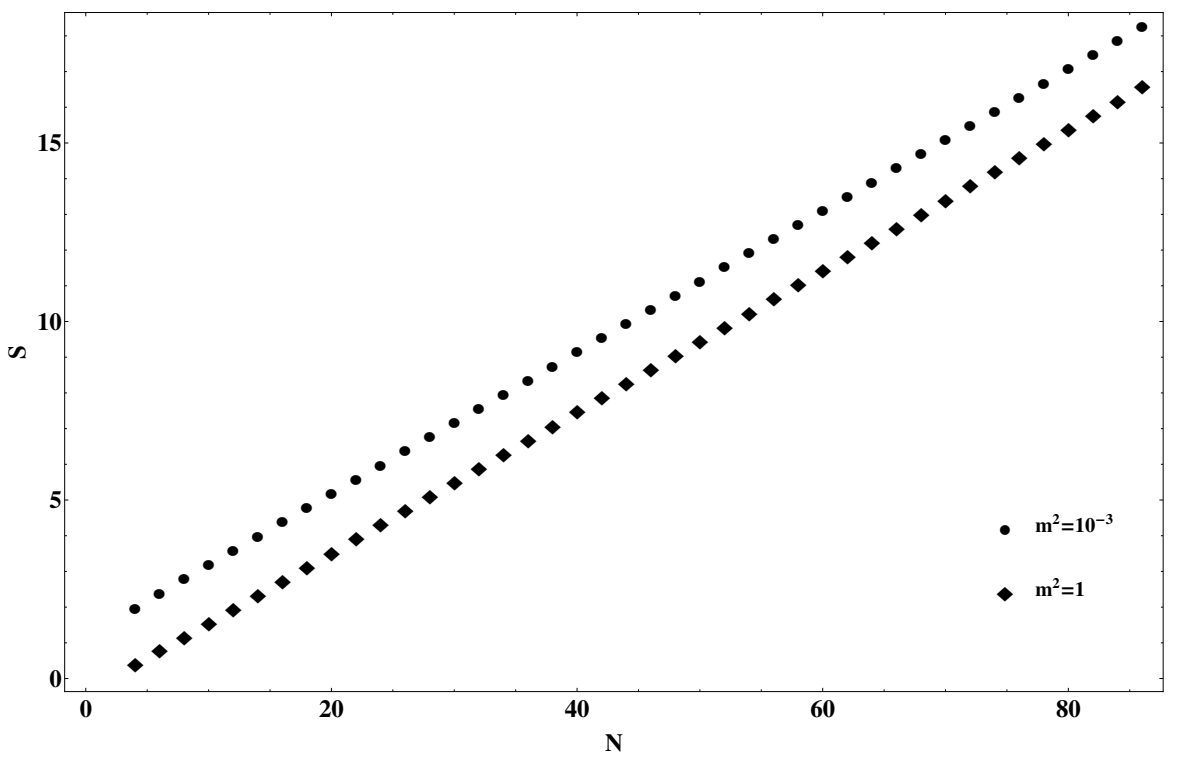

Figure 3. Entanglement entropy for half the sphere as a function of $\mathrm{N}$ for $m^{2}=1$ and $m^{2}=0.001$. Both lines have a slope of 0.2 .

mass), leading us to conclude that

$$
\frac{S}{N}=F(\theta)+\mathcal{O}\left(N^{-1}\right) \text { corrections }
$$

where $F(\theta)$ is proportional to $\theta^{2}$ for small $\theta$, is smooth for $\theta \in[0, \pi]$ and has the property that $F(\pi-\theta)=F(\theta)$.

The claim in equation (3.5) is further supported by figure 4 , which shows $S / N$ as a function of the fractional area of the polar cap,

$$
a:=\frac{\operatorname{area}(C)}{4 \pi R^{2}}=\sin ^{2}(\theta / 2),
$$

for several different values of $N$. The convergence to a fixed curve at large $N$ is evident.

Result (3.5) is quite reasonable from the point of view of the matrix model. We can think of the square matrix in figure 1 as literally a square block of coupled harmonic oscillators. In this way of thinking, the couplings arising from Hamiltonian (1.5) are only among nearest-neighbour oscillators. Therefore, we would expect the entanglement entropy to follow an area law. The length of the boundary is the number of matrix elements laying on the diagonal in figure 1 , which is $2 j(1-\cos \theta)=2 N a$, thus we expect the entanglement entropy in this square array of oscillators to be proportional to $N a$, which is what we see for small $a$ in figure 4 .

Finally, we studied dependence of the entanglement entropy on the dimensionless mass $m$ of the field. Figure 5 shows the entanglement entropy for half the sphere as a function of $m^{-1}$. Over a wide range of masses, for $m<N$, the entanglement entropy appears approximately independent of the mass. This is the region in which our result (3.5) is applicable. In fact, in this region, $S$ must slowly raise with $m^{-1}$, since at $m=0$ the 


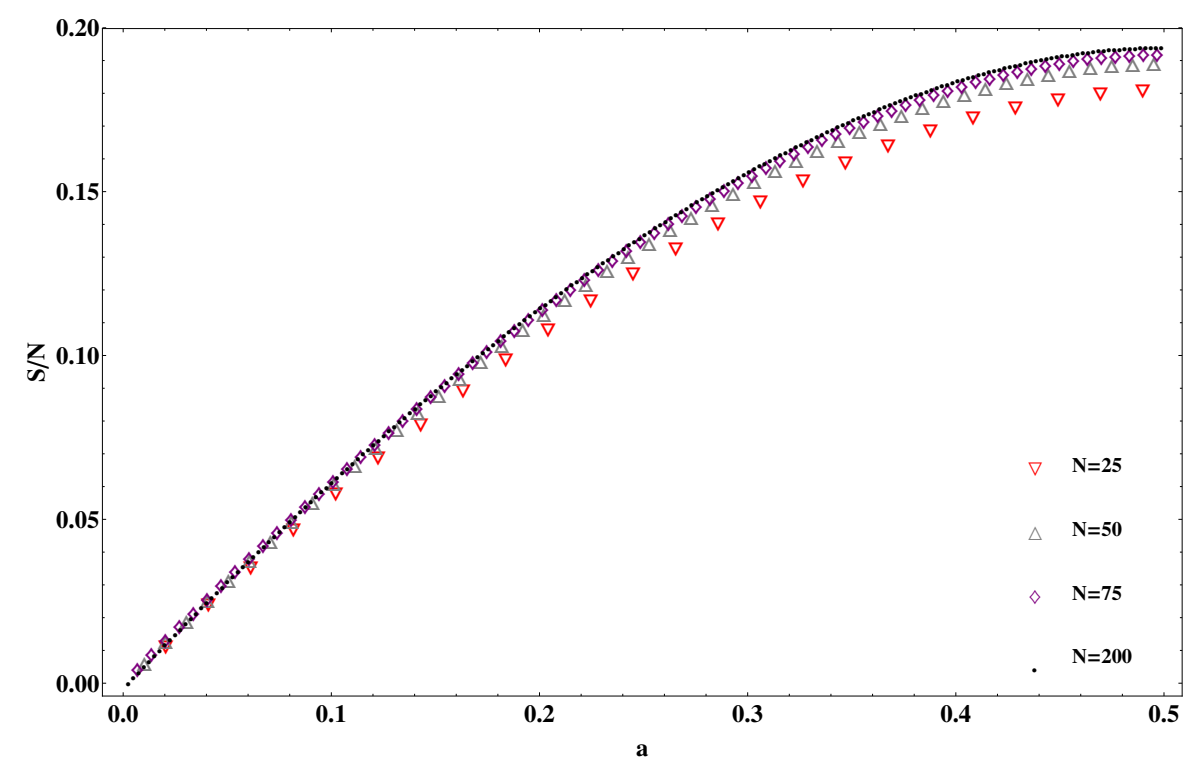

Figure 4. Entanglement entropy $S$ scaled by $N$ as the fractional area $a$ of polar cap for different values of $N$. Notice that $S / N$ converges to a good large $N$ limit. For small $\theta S / N$ appears proportional to $C$, consistent with an extensive entanglement entropy. $m=1$.

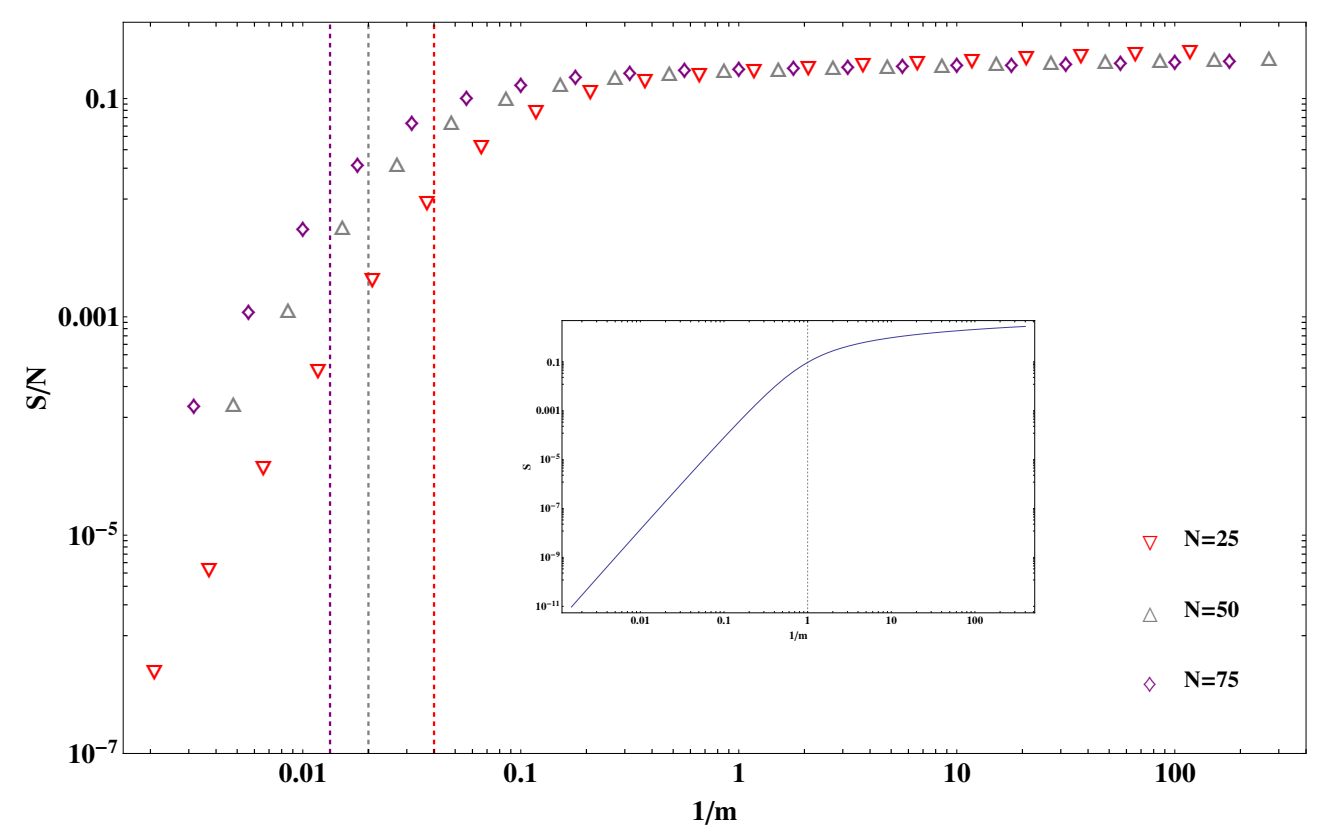

Figure 5. Entanglement entropy for half the sphere as a function of inverse mass. Vertical lines indicate mass at which the diagonal and off-diagonal elements of the Hamiltonian (1.5) matrix are of the same order $(m \sim N)$. The inset shows entanglement entropy for two coupled harmonic oscillators whose potential energy is given by equation (3.7). 


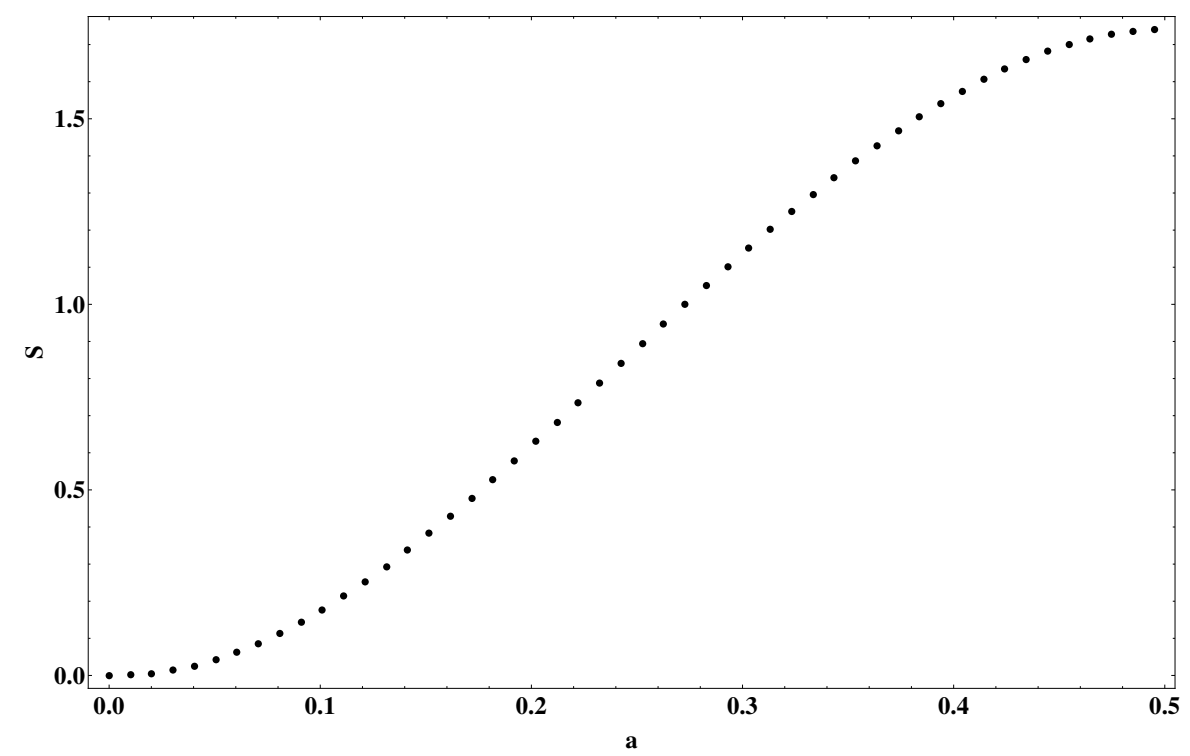

Figure 6. Entanglement entropy $S$ scaled by $N$ as the fractional area $a$ of polar cap for a large mass, $m^{2}=1000(N=50)$.

entropy is infinite due to the appearance of a flat direction in the model. For large masses $m>N$, the entropy decreases to zero, as the kinetic term (which couples degrees of freedom at different points and is the source of entanglement entropy) is overwhelmed by the mass term (which does not couple degrees of freedom at different points). To understand this behaviour, let's consider a toy model of two coupled oscillators $x_{1}$ and $x_{2}$ (of equal masses) coupled by a potential

$$
\left(1+m^{2}\right) x_{1}^{2}+\left(1+m^{2}\right) x_{2}^{2}+2 x_{1} x_{2} .
$$

Entanglement entropy for one of these oscillators, in the vacuum of the system and as a function of $m^{-1}$ is shown in the inset to figure 5. This toy example models the full Hamiltonian (1.5): the kinetic term in (1.5) contributes diagonal and off-diagonal terms (of order 1 in the toy Hamiltonian (3.7)), while the mass term contributes only diagonal terms. We see that the behaviour of the entanglement entropy in this toy model has the same qualitative features as the entanglement entropy on the sphere.

When we study entanglement entropy for a field with a large mass, we find that it grows with $N$ faster than linear, and that its behaviour as a function of $\theta$ at small $\theta$ and at fixed $N$ is $\theta^{a}$, with a power $a>2$ (figure 6). Notice that in the limit of infinite mass, the harmonic oscillators that make up our noncommutative sphere appear effectively uncoupled, so it is not surprising that entanglement entropy is smaller at a large mass. We leave an exploration of the details of this behaviour to future work.

\section{Discussion and future work}

An interesting point about our construction of a region in noncommutative geometry, which is illustrated in figure 1, is that the number of oscillators in the triangular corner of the 


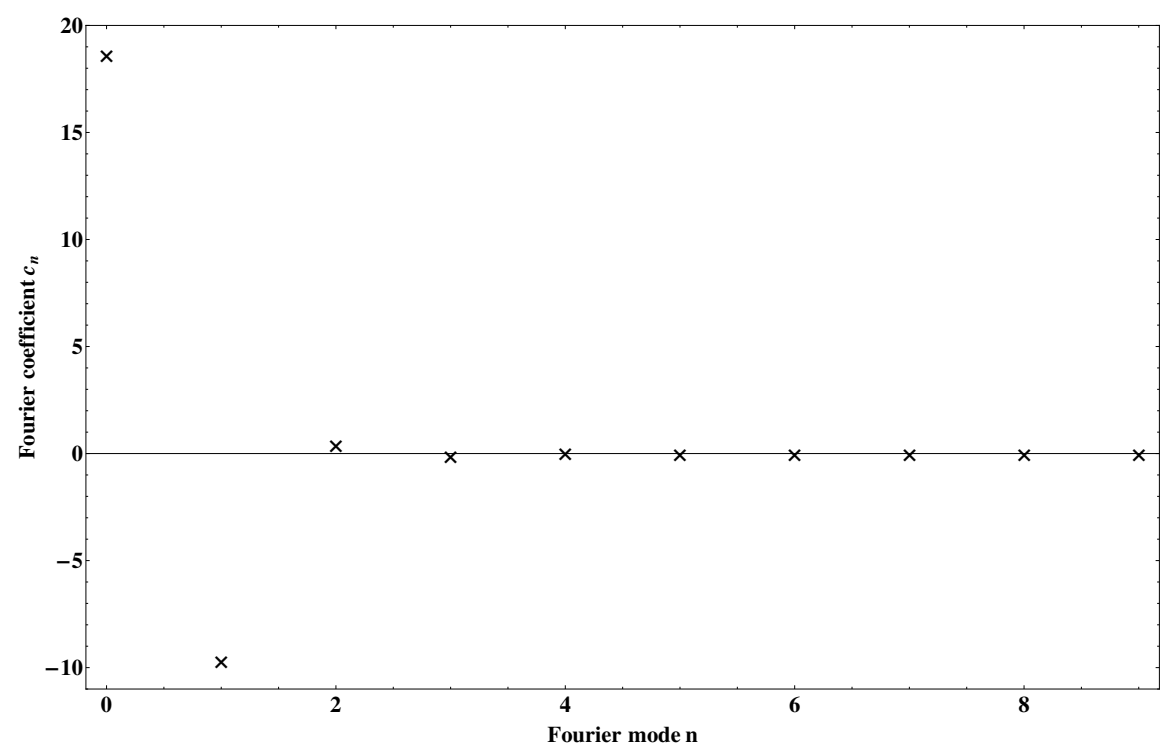

Figure 7. Coefficients of $\cos (2 n \theta)$ terms in a Fourier expansion for entanglement entropy $S$ shown in figure 2. Since entanglement entropy is symmetric about $\theta=\frac{\pi}{2}$, the Fourier coefficients $c_{n}$ satisfy $c_{n}=c_{400-n}$ for $n=1 \ldots 199$, which implies $S(\theta)=c_{0}+2 \sum_{n=1}^{199} c_{n} \cos (2 n \theta)+c_{200} \cos (2 \cdot 200 \cdot \theta)$.

matrix is approximately $2 j^{2}(1-\cos \theta)^{2}$ or $2 N^{2} a^{2}$. Thus, this number of degrees of freedom associated with a polar cap does not grow proportionately to its fractional area $a$, but rather to the square of $a$. This would not be possible in a local theory (where degrees of freedom are more-or-less associated with points in the underlying geometry). It also implies that there is no reason why the degrees of freedom associated with a subregion of the sphere should be uniformly distributed within this subregion. If degrees of freedom in a subregion of a noncommutative sphere reside predominantly near the boundary of the region, we can account for our entanglement entropy results as follows: just like in commutative theories, quantum correlations between a region and its complement develop only across the boundary, but since these degrees of freedom are concentrated near the boundary, entanglement entropy grows more rapidly than it would in a commutative theory. Such a 'reshuffling' of degrees of freedom when a finite region is considered would be an interesting way in which a noncommutative field theory can reproduce the entanglement entropy predicted by holography.

An alternative interpretation of our results can be obtained when we realize that our result for the entanglement entropy can be written in some suggestive ways. In the linear regime, we have that

$$
S=\kappa N \frac{\operatorname{area}(C)}{R^{2}}=\kappa \frac{\operatorname{area}(C)}{\boldsymbol{\theta}} .
$$

This should be compared with the general form of the result from [8], which was

$$
S \sim \frac{\operatorname{area}(C)}{\epsilon^{2}} .
$$

It is as if the UV cutoff $\epsilon$ was replaced by $\sqrt{\boldsymbol{\theta}}$. Such a replacement would be fairly natural in 
the case of area-law entropy, as $\sqrt{\boldsymbol{\theta}}$ is the thickness of the boundary; however, it is hard to see how the fuzziness of a boundary definition can affect entropy in the volume-law regime. It would be very interesting to see what happens to the entanglement entropy if one can define a region with a boundary whose thickness is less than $\sqrt{\boldsymbol{\theta}}$, ideally as small as $\epsilon$.

A further intriguing fact about the entanglement entropy we have found is that $F(\theta)$ in equation (3.5) is very close to a multiple of $\sin ^{2}(\theta)$. This is presented in figure 7 ; the first three coefficients are 18.6, -9.68 and 0.443. The ratio of the first two coefficients is $-1.92 \approx-2$, which leads to $F(\theta) \sim \sin ^{2}(\theta)=a(1-a)$, where $a=\sin ^{2}(\theta / 2)$ is the fractional area of the polar cap. The expression

$$
S \approx N \kappa a(1-a), \quad \kappa=0.8
$$

is most suggestive. If degrees of freedom are pictured as uniformly distributed on the sphere, the dependence of $S$ on the area $a$ is most easily explained by assuming that every (localized) degree of freedom on the sphere has its entanglement uniformly spread over the whole sphere. This is in sharp contrast with local theories, where short-ranged interactions lead to quantum correlations between a region and its complement being established across the boundary surface, leading to the area-law for entanglement entropy [23]. In this interpretation, however, it is puzzling why the entanglement entropy does not grow like the total number of degrees of freedom, $N^{2}$. Perhaps the answer is that the localized degrees of freedom are only weakly entangled with each other. Or perhaps the $N$-dependence is caused by the thickness of the boundary (notice that replacing $N$ with $N^{2}$ in equation (4.3) is equivalent to changing $\boldsymbol{\theta}$ to $\epsilon^{2}$ in equation (4.1), which turns it into equation (4.2)).

To distinguish between these different interpretations of our results, further study is needed. In addition to finding a procedure for building regions with thinner boundaries, one could also study mutual information. Since mutual information is UV finite in local theories, it might have a different dependence on the UV and other cutoffs in the noncommutative theory.

Our method for obtaining finite cap region on the noncommutative sphere can also be applied to disk-like regions in the noncommutative plane. It would be interesting to study entanglement entropy in that geometry, (necessarily) for an interacting field theory, and to see whether entanglement entropy in a field theory really does have a phase transition at a length-scale $\boldsymbol{\theta} / \epsilon$, in accordance with holographic results. Moreover, the theory on a noncommutative plane is related to the quantum Hall effect [24]. Other geometries that would be interesting to study would be higher dimensional spheres as well as noncommutative tori in different dimensions.

\section{Acknowledgments}

We are grateful for helpful discussions with Charles Rabideau, Gordon Semenoff, Mark van Raamsdonk and Ken Yeh. This work was completed with support from the Natural Sciences and Engineering Council of Canada (NSERC) and from the Fonds de recherche du Québec - Nature et technologies (FRQNT). 
Open Access. This article is distributed under the terms of the Creative Commons Attribution License (CC-BY 4.0), which permits any use, distribution and reproduction in any medium, provided the original author(s) and source are credited.

\section{References}

[1] Y. Sekino and L. Susskind, Fast scramblers, JHEP 10 (2008) 065 [arXiv:0808.2096] [INSPIRE].

[2] M. Edalati, W. Fischler, J.F. Pedraza and W. Tangarife Garcia, Fast scramblers and non-commutative gauge theories, JHEP 07 (2012) 043 [arXiv:1204.5748] [INSPIRE].

[3] N. Seiberg and E. Witten, String theory and noncommutative geometry, JHEP 09 (1999) 032 [hep-th/9908142] [INSPIRE].

[4] A. Hashimoto and N. Itzhaki, Noncommutative Yang-Mills and the AdS/CFT correspondence, Phys. Lett. B 465 (1999) 142 [hep-th/9907166] [INSPIRE].

[5] J.M. Maldacena and J.G. Russo, Large-N limit of noncommutative gauge theories, JHEP 09 (1999) 025 [hep-th/9908134] [INSPIRE].

[6] N. Lashkari, Equilibration of small and large subsystems in field theories and matrix models, arXiv:1304.6416 [INSPIRE].

[7] W. Fischler, A. Kundu and S. Kundu, Holographic entanglement in a noncommutative gauge theory, JHEP 01 (2014) 137 [arXiv:1307.2932] [INSPIRE].

[8] J.L. Karczmarek and C. Rabideau, Holographic entanglement entropy in nonlocal theories, arXiv: 1307.3517 [INSPIRE].

[9] J.L. Barbon and C.A. Fuertes, Holographic entanglement entropy probes (non)locality, JHEP 04 (2008) 096 [arXiv: 0803.1928] [INSPIRE].

[10] D. Dou and B. Ydri, Entanglement entropy on fuzzy spaces, Phys. Rev. D 74 (2006) 044014 [gr-qc/0605003] [INSPIRE].

[11] D. Dou, Comments on the entanglement entropy on fuzzy spaces, Mod. Phys. Lett. A 24 (2009) 2467 [arXiv: 0903.3731] [INSPIRE].

[12] J. Madore, The fuzzy sphere, Class. Quant. Grav. 9 (1992) 69 [inSPIRE].

[13] M.R. Douglas and N.A. Nekrasov, Noncommutative field theory, Rev. Mod. Phys. 73 (2001) 977 [hep-th/0106048] [INSPIRE].

[14] S. Minwalla, M. Van Raamsdonk and N. Seiberg, Noncommutative perturbative dynamics, JHEP 02 (2000) 020 [hep-th/9912072] [INSPIRE].

[15] C.-S. Chu, J. Madore and H. Steinacker, Scaling limits of the fuzzy sphere at one loop, JHEP 08 (2001) 038 [hep-th/0106205] [INSPIRE].

[16] P. Castro-Villarreal, R. Delgadillo-Blando and B. Ydri, A gauge-invariant UV-IR mixing and the corresponding phase transition for $\mathrm{U}(1)$ fields on the fuzzy sphere, Nucl. Phys. B $\mathbf{7 0 4}$ (2005) 111 [hep-th/0405201] [INSPIRE].

[17] G. Alexanian, A. Pinzul and A. Stern, Generalized coherent state approach to star products and applications to the fuzzy sphere, Nucl. Phys. B 600 (2001) 531 [hep-th/0010187] [INSPIRE].

[18] F. Berezin, General concept of quantization, Commun. Math. Phys. 40 (1975) 153. 
[19] A. Hammou, M. Lagraa and M. Sheikh-Jabbari, Coherent state induced star product on $R_{\lambda}^{3}$ and the fuzzy sphere, Phys. Rev. D 66 (2002) 025025 [hep-th/0110291] [INSPIRE].

[20] J.J. Sakurai and J. Napolitano, Modern quantum mechanics, Addison Wesley (1993).

[21] P. Prešnajder, The Origin of chiral anomaly and the noncommutative geometry, J. Math. Phys. 41 (2000) 2789 [hep-th/9912050] [INSPIRE].

[22] M. Srednicki, Entropy and area, Phys. Rev. Lett. 71 (1993) 666 [hep-th/9303048] [INSPIRE].

[23] J. Eisert, M. Cramer and M. Plenio, Area laws for the entanglement entropy - a review, Rev. Mod. Phys. 82 (2010) 277 [arXiv:0808.3773] [INSPIRE].

[24] D. Bigatti and L. Susskind, Magnetic fields, branes and noncommutative geometry, Phys. Rev. D 62 (2000) 066004 [hep-th/9908056] [INSPIRE]. 